Article

\title{
Synthesis and Physical Properties of Non-Crystalline Nylon 6 Containing Dimer Acid
}

\author{
Ching-Nan Huang ${ }^{1}$, Chang-Mou Wu ${ }^{2} \oplus$, Hao-Wen Lo ${ }^{1}$, Chiu-Chun Lai ${ }^{1, *}$, Wei-Feng Teng ${ }^{3}$, \\ Lung-Chang Liu ${ }^{3}$ and Chien-Ming Chen ${ }^{3}$ \\ 1 Department of Textile Engineering, Chinese Culture University, Taipei 11114, Taiwan; \\ chingnanhuang57@yahoo.com (C.-N.H.); deanwillmadeit2236@gmail.com (H.-W.L.) \\ 2 Department of Materials Science and Engineering, National Taiwan University of Science and Technology, \\ Taipei 10607, Taiwan; cmwu@mail.ntust.edu.tw \\ 3 Material and Chemical Research Laboratories, Industrial Technology Research Institute, \\ Hsinchu 30011, Taiwan; wfteng@yahoo.com (W.-F.T.); markloukimo@yahoo.com.tw (L.-C.L.); \\ cmchen101@yahoo.com (C.-M.C.) \\ * Correspondence: lqj2@ulive.pccu.edu.tw
}

Received: 23 December 2018; Accepted: 14 February 2019; Published: 25 February 2019

\begin{abstract}
In this study, a long carbon chain dimer acid is introduced into a nylon 6 structure and is copolymerized with different structural amines to produce amorphous nylon 6 by 4,4'-methylenebis(2-methylcyclohexylamine) (MMCA) in different copolymerization ratios. The effect of different structures and copolymerization ratios on the properties of nylon 6 is determined, along with the thermal properties, crystallinity, water absorption, dynamic mechanical properties, and optical properties. It is found that the melting point and the thermal cracking temperature Td10 of nylon 6 are respectively between $176{ }^{\circ} \mathrm{C}$ and $213{ }^{\circ} \mathrm{C}$ and $378{ }^{\circ} \mathrm{C}$ to $405{ }^{\circ} \mathrm{C}$. The effect of introducing a bicyclohexane group containing a methyl side chain is greater than that of a meta-benzene ring, so COMM (synthesized by Caprolactam (C), dimer oleic acid (OA), and 4, $4^{\prime}$-Methylenebis(2-methylcyclohexylamine) (MMCA)) has the lowest melting point, enthalpy, and crystallinity. As the copolymerization ratio increases, its thermal properties decrease. 10\% is the lowest crystallinity. The amine structure containing a bicycloalkyl group has lower water absorption and a $10 \%$ copolymerization ratio gives the lowest water absorption. It contains the bicycloalkyl group, COM (synthesized by Caprolactam (C), dimer oleic acid (OA) and 4, $4^{\prime}$-Methylenebis(cyclohexylamine) (MCA)), which has the highest loss modulus. The lowest loss modulus is noted for a copolymerization ratio of $7 \%$ and the value of $\tan \delta$ increases as the copolymerization ratio increases. The introduction of nylon 6 with the bicycloalkyl groups, COMM and COM, significantly increases transparency. As the copolymerization ratio increases, the transparency increases and the haze decreases. The best optical properties are achieved for $10 \%$ copolymerization.
\end{abstract}

Keywords: amphours nylon 6; transparency; ring-opening polymerization

\section{Introduction}

Nylon 6 prepared by ring-opening polymerization of caprolactam is a common engineering polymer material. It has excellent properties, such as good chemical resistance, high mechanical properties, and high thermal stability. It is easily processed and is has been widely used in automotive, textile, packaging, biomedical applications, and solar cells. Although nylon 6 has excellent mechanical properties, good heat resistance, wear resistance and chemical solvent, and is very easily processed, the structure has a mercapto group so the hydrophilic group easily generates hydrogen bonds with 
water molecules, which means that nylon 6 readily absorbs water. Water absorption increases as the density of the amide group in the molecular structure increases. Dimensional changes occur when the material absorbs water, so the dimensional stability of the product is reduced. The tensile strength and bending strength decrease as the moisture absorption rate increases, which affects other properties of the product $[1,2]$.

Generally, high molecular weight polymers contain crystalline and amorphous elements. The degree of crystallinity has a significant influence on the properties of high molecular weight polymers. Since the indoleamine groups in the nylon 6 molecular chain are arranged neatly, the intermolecular force is strong and the crystallinity is high. However, the high crystallinity of nylon 6 results in low transparency and high haze, limiting its applications in optical films, electrical devices and food packaging. The demand for high optical transparency polymers has increased in recent years. Therefore, the improvement of water absorption, transparency and haze for nylon 6 has become important for its applications in optical films and food packaging [3]. In this study, non-crystalline nylon 6 is prepared by copolymerization of dimer acid with different structural amines and different proportions of amines. Its thermal properties, optical transparency and hygroscopic characteristics are determined.

\section{Materials and Methods}

\subsection{Materials}

H: Hexanediamine (HMDA), C: 1,3-Cyclohexanebis(methylamine) (CHMA), M:4,4'Methylenebis(cyclohexylamine) (MCA), I:Isophorondiamine (IDPA), MM: 4,4'-Methylenebis(2methylcyclohexylamine) (MMCA), X: m-Xylylenediamine (XDA) were obtained from Acros (New Taipei City, Taiwan). These were purified by distillation. C: Caprolactam(CPL) and O: dimer oleic acid(OA)were obtained from commercial sources and purified by recrystallization.

\subsection{Nylon Salts}

The salt can be prepared by mixing the water or alcoholic solutions of the two components because of different hydrophobic property. Nylon salts were prepared by adding a $70 \%$ water solution of diamine to a $25 \%$ alcoholic solution of diacid at $60^{\circ} \mathrm{C}$ and regulated the $\mathrm{pH}$ value to 7.5 . The solutions of salt were clarified by active carbon powder at room temperature. The aqueous salt solutions were concentrated of about $50-60 \%$.

\subsection{Polymerization}

The solution of salts proceeds polymerization of condensation. A $60 \%$ solution of each salt and Caprolactam(CPL) were heated for $3 \mathrm{~h}$ from temperature 30 to $220{ }^{\circ} \mathrm{C}$ in autoclave before prepolymerization of $2 \mathrm{~h}$ at $220^{\circ} \mathrm{C}$ and atmospheric pressure at nitrogen atmosphere. The polymerization temperature was $260^{\circ} \mathrm{C}$ and reaction $4 \mathrm{~h}$ until a specific viscosity was achieved shows in Figure 1. The different amines and acid should take different polymerization time 2-4 h [4]. The relative viscosity (RV) were measured with a Brookfield KV100 capillary viscometer at $25^{\circ} \mathrm{C}$.

\subsection{Preparation of Films}

The film samples were made by injection molding at temperatures of 230 to $260{ }^{\circ} \mathrm{C}$ above their melting points. The samples were maintained in a vacuum to prevent oxidation.

\subsection{Thermal Analysis}

Using Perkin-Elmer thermal analysis facilities proceed Differential Scanning Calorimerty (DSC), Thermogravimertic Analyzers (TGA) and Dynamic Mechanical Analysis (DMA) analysis. The samples were dehydrated to $800 \mathrm{ppm}$ water content. DSC sample $10 \mathrm{mg}$ of each film was placed on platinum sample holder at a heating rate of $10^{\circ} \mathrm{C} / \mathrm{min}$ under flowing nitrogen. The testing temperature was 
between room temperature to $250^{\circ} \mathrm{C}$. TGA sample $5 \mathrm{mg}$ of each film was placed on platinum sample holder at a heating rate of $20^{\circ} \mathrm{C} / \mathrm{min}$ under flowing nitrogen. The testing temperature was between room temperature to $400{ }^{\circ} \mathrm{C}$. DMA sample $10 \mathrm{mg}$ of each film was placed on platinum sample holder at a heating rate of $10^{\circ} \mathrm{C} / \mathrm{min}$ under flowing nitrogen. The testing temperature was between -40 to $140{ }^{\circ} \mathrm{C}$.

\subsection{Sorption Isotherm}

The samples that were used to determine the sorption properties were $10 \mathrm{~mm}$ square. These were immersed in deionized water at $28^{\circ} \mathrm{C}$ for $24 \mathrm{~h}$ and $48 \mathrm{~h}$. The weight was measured to calculate the absorption rate.

\subsection{X-ray Diffraction}

The X-ray diffraction patterns for the samples were obtained using a Bruker D8Advance diffractometer (Bruker, Hsinchu city, Taiwan) with an acceleration voltage of $40 \mathrm{kV}$ and a current of $30 \mathrm{~mA}$. Data was collected at room temperature in the $2 \theta$ range from $3^{\circ}$ to $50^{\circ}$.

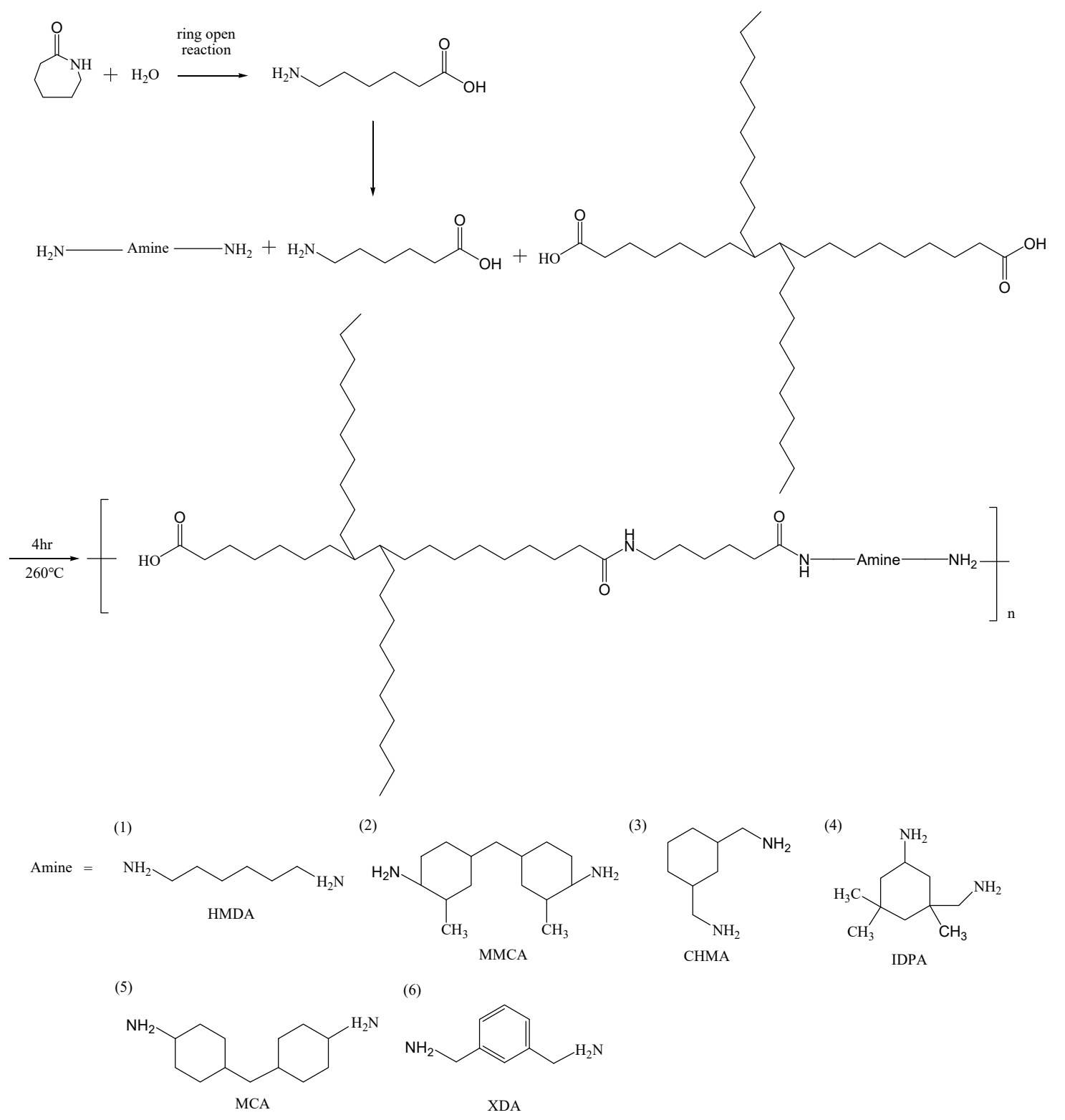

Figure 1. Synthesis of PA6 containing cyclic compounds. 


\subsection{Optical properties}

Based on ASTM D1003-61 (1997) method (Standard Test Method for Haze and Luminous Transmittance of Transparent Plastics), haze and transparency were obtained by haze meter (Labsanli WGT-S). The standard light were A $(2856 \mathrm{~K})$ and $\mathrm{C}(6447 \mathrm{~K})$ at condition was $23+2{ }^{\circ} \mathrm{C}\left(73.4+3.6^{\circ} \mathrm{F}\right)$ and RH $50 \%+5 \%$. The thickness of sample was $15 \mathrm{~mm}$.

$$
\text { Haze }=T \mathrm{~d} / T \mathrm{t} \times 100
$$

$T d$ : the transmittance of sample, Tt: the total transmittance.

\section{Results and Discussion}

\subsection{Identification}

The functional group of the synthesized product was confirmed by Fourier Transform Infrared Spectroscopy (FT-IR) between 4000 and $550 \mathrm{~cm}^{-1}$, in order to determine the molecular structure of the synthesized product. Figure 2 shows the FTIR spectrum for nylon 6 with different copolymerization components. The nylon 6 peaks for different copolymerization components appear as the $\mathrm{N}-\mathrm{H}$ stretching vibration peaks at $3300 \mathrm{~cm}^{-1}$, the $\mathrm{C}=\mathrm{O}$ stretching vibration peak at $1650 \mathrm{~cm}^{-1}$ and the $\mathrm{C}-\mathrm{N}-\mathrm{H}$ bending and the $\mathrm{C}-\mathrm{N}$ stretching vibration peaks at approximately $1500 \sim 1530 \mathrm{~cm}^{-1}$. The COX shows a vibration peak for the benzene ring at $750-800 \mathrm{~cm}^{-1}$.

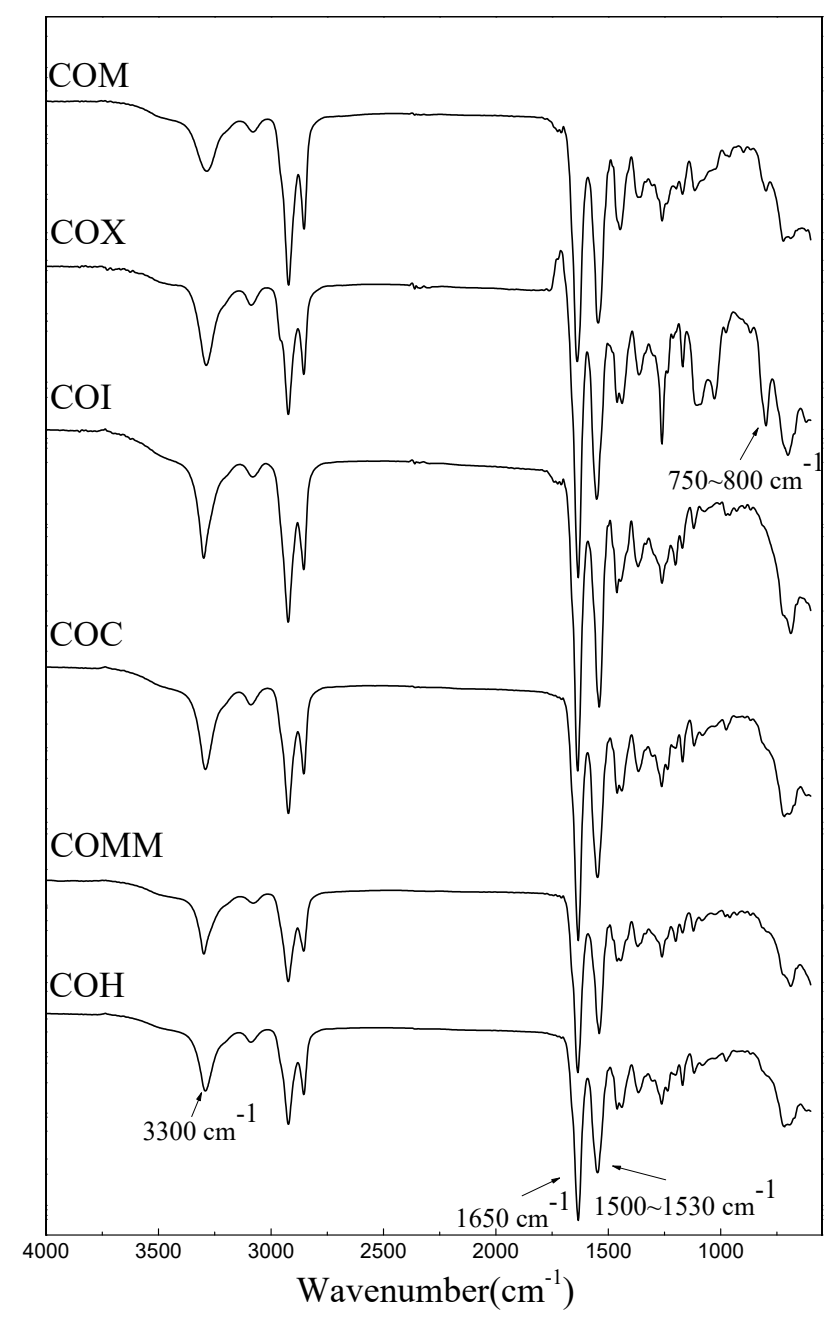

Figure 2. FTIR spectrum of PA6 containing cyclic compounds. 


\subsection{PA6-containing Cyclic Compounds}

This study determines the physical effect of different types of amine PA6 copolymerization. The code for the composition, the composition of the RV and the amine group are shown in Table 1.

Table 1. Composition of PA6 containing cyclic compounds.

\begin{tabular}{cccccccc}
\hline Polymer & $\begin{array}{c}\text { PA6 } \\
\text { molar ratio }\end{array}$ & $\begin{array}{c}\text { ACID } \\
\text { molar ratio }\end{array}$ & $\begin{array}{c}\text { BASE } \\
\text { molar ratio }\end{array}$ & RV & $\begin{array}{c}\text { NH2 } \\
\text { meq/kg }\end{array}$ & $\boldsymbol{M}_{\mathbf{w}}$ & $\boldsymbol{M}_{\mathbf{w}} / \boldsymbol{M}_{\mathbf{n}}$ \\
\hline COM & CPL, 80\% & OA, 10\% & MCA, 10\% & 2.10 & 63 & 34556 & 1.42 \\
COX & CPL, 80\% & OA, 10\% & XDA, 10\% & 2.31 & 63 & 32478 & 1.56 \\
COC & CPL, 80\% & OA, 10\% & CHMA, 10\% & 2.05 & 83 & 34558 & 1.48 \\
COH & CPL, 80\% & OA, 10\% & HMDA, 10\% & 2.44 & 66 & 38774 & 1.33 \\
COI & CPL, 80\% & OA, 10\% & IDPA, 10\% & 2.25 & 60 & 32879 & 1.59 \\
COMM & CPL, $80 \%$ & OA, 10\% & MMCA, 10\% & 2.13 & 64 & 34226 & 1.49 \\
\hline
\end{tabular}

C: Caprolactam(CPL), O: dimer oleic acid (OA), H: Hexanediamine (HMDA), C: 1,3-Cyclohexanebis(methylamine) (CHMA), M: 4,4'-Methylenebis(cyclohexylamine) (MCA), I:Isophorondiamine (IDPA), MM:4,4'-Methylenebis(2methylcyclohexylamine) (MMCA), X: m-Xylylenediamine (XDA).

\subsubsection{Thermal Properties}

Figure 3 shows a TGA diagram for a COC nylon 6 copolymer under nitrogen gas. The respective values for $T \mathrm{~d} 10$ and $T \mathrm{~d} 50$ are 405 and $444^{\circ} \mathrm{C}$. The $T \mathrm{~d} 10$ and $T \mathrm{~d} 50$ values for each polymer are listed in Table 2. It is seen that $\mathrm{Td} 10$ is between 378 and $405^{\circ} \mathrm{C}$ and $\mathrm{Td} 50$ is between 431 and $444{ }^{\circ} \mathrm{C}$. COMM has the lowest value for $\mathrm{Td} 10$. Each polymer experiences thermal weight loss at more than $30^{\circ} \mathrm{C}$. It is also confirmed that each of the polymers has good heat resistance properties.

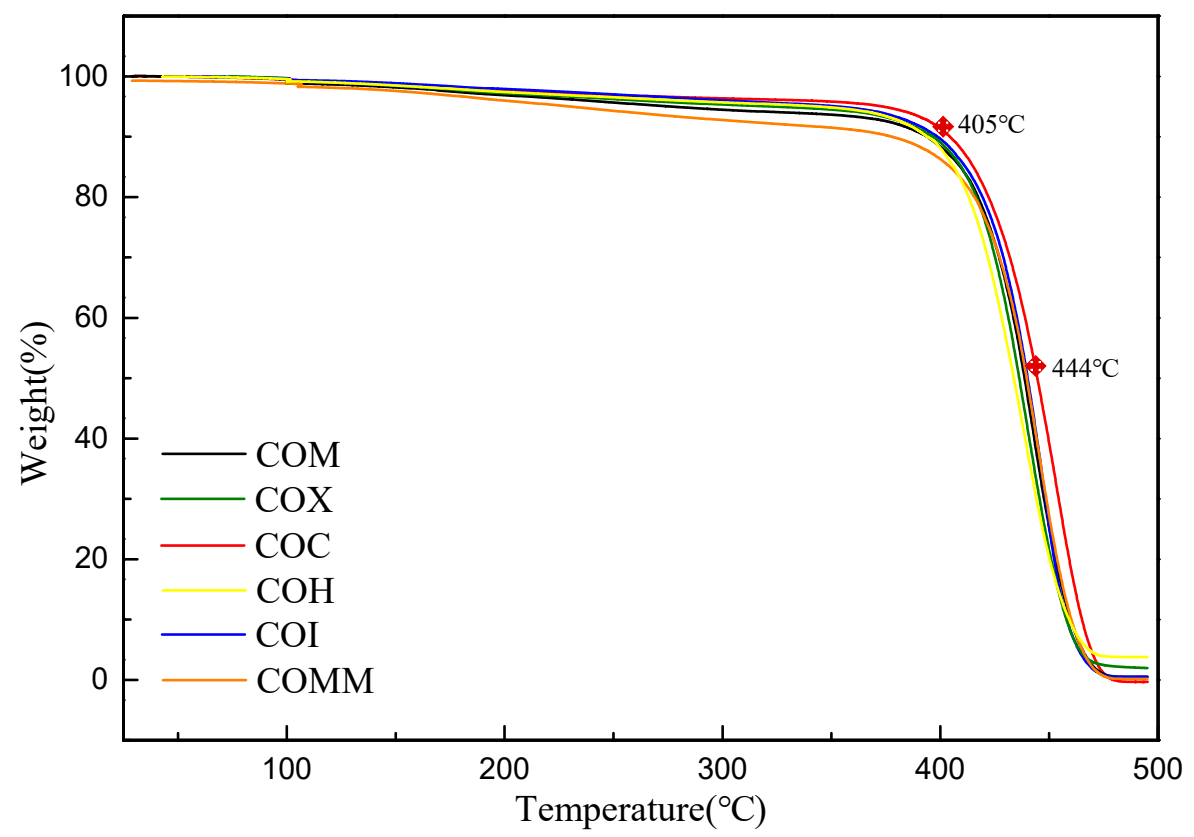

Figure 3. TGA Of PA6 containing cyclic compounds.

The melting point, enthalpy and crystallinity of the synthetic products were measured using DSC, Figure 4 shows the DSC chart for different copolymerized nylon 6 samples. The $T_{\mathrm{m}}$ and crystallinity for each polymer are listed in Table 2. In terms of melting point, the main component of the polymer is nylon 6 . The copolymerization of oleic acid dimer with $10 \%$ proportional long carbon chain and amines with different structures affects the melting point of nylon 6, so the melting point of the polymer is between 178 and $191{ }^{\circ} \mathrm{C}$. In terms of crystallinity, the introduction of a bicyclohexane group 
containing a methyl side chain has a greater effect than a metacyclic benzene ring. COMM has the lowest crystallinity because its bulky structure disturbs the polymer molecular chain.

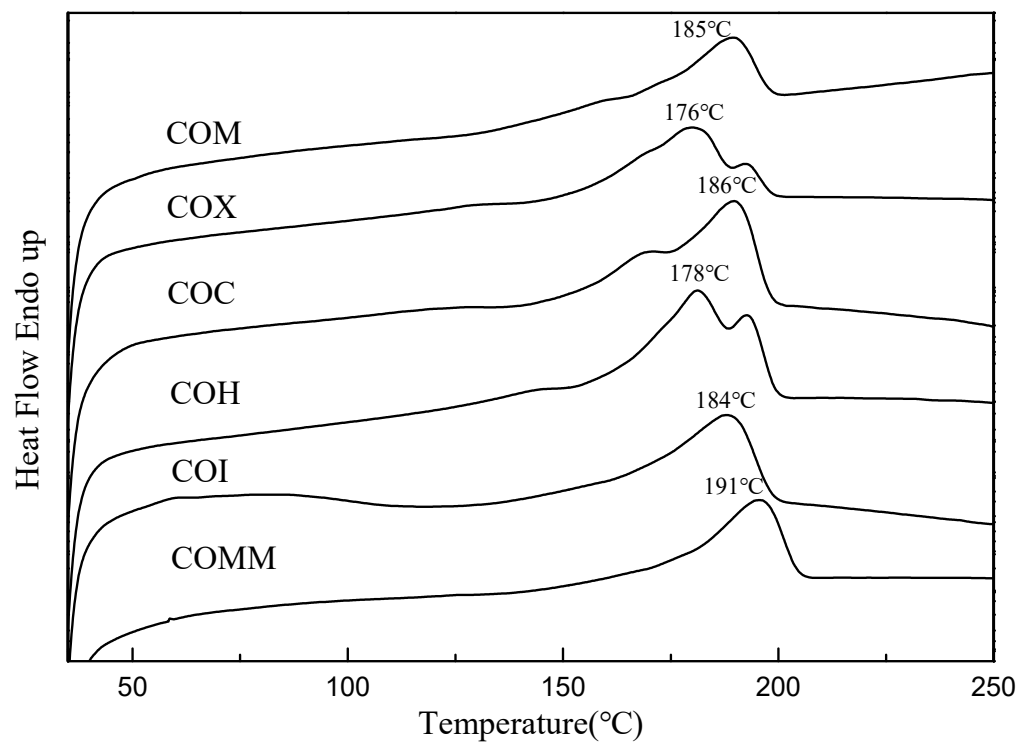

Figure 4. DSC for PA6 containing cyclic compounds.

Table 2. Thermal properties of PA6 containing cyclic compounds.

\begin{tabular}{ccccccc}
\hline Polymer & $\begin{array}{c}\boldsymbol{T}_{\mathbf{g}} \\
{ }^{\circ} \mathbf{C}\end{array}$ & $\begin{array}{c}\boldsymbol{T}_{\mathbf{m}} \\
{ }^{\circ} \mathbf{C}\end{array}$ & $\begin{array}{c}\boldsymbol{\Delta} \boldsymbol{H} \\
\mathbf{J} / \mathbf{g}\end{array}$ & $\begin{array}{c}\text { Crystallinity } \\
\mathbf{\%}\end{array}$ & $\begin{array}{c}\mathbf{T}_{\mathbf{d}} \mathbf{1 0} \\
{ }^{\circ} \mathbf{C}\end{array}$ & $\begin{array}{c}\mathbf{T}_{\mathbf{d}} \mathbf{5 0} \\
{ }^{\circ} \mathbf{C}\end{array}$ \\
\hline COM & 32.8 & 185 & 24.0 & 10.0 & 395 & 438 \\
COX & 25.9 & 176 & 26.7 & 11.1 & 398 & 436 \\
COC & 30.1 & 186 & 23.4 & 9.7 & 405 & 444 \\
COH & 19.4 & 178 & 33.8 & 14.0 & 394 & 431 \\
COI & 34.7 & 184 & 23.8 & 9.9 & 397 & 440 \\
COMM & 34.2 & 191 & 21.6 & 9.0 & 378 & 439
\end{tabular}

$T_{\mathrm{g}}$ : glass transition measured by DMA, Crystallinity: the value of $100 \%$ crystalline material's melting heat is 240 .

This study uses a dynamic mechanical analyzer (DMA) to determine the storage modulus, the loss modulus and the $\tan \delta$ for the synthesized product. The data shows the strength, viscosity, elasticity and glass transition temperature of the material as a function of temperature. Figure 5 shows the loss modulus diagram for nylon 6 with different copolymerization components. The figure shows that as the temperature rises close to its $T_{\mathrm{g}}$ point, the material becomes soft and the loss modulus gradually increases. The bicycloalkyl group containing COM has the highest loss modulus, mainly due to the absence of methyl side chains and meta benzene rings in the structure. The cycloalkane-containing methyl group and the meta-phenylene ring nylon 6 interfere with the movement of the molecular chain, resulting in a lower loss modulus. Figure 6 shows the tan $\delta$ value for nylon 6 with different copolymerization components. COMM and COI containing methyl side chains have a greater influence on nylon 6 and are very rigid, so the value of $T_{\mathrm{g}}$ is higher [5-7]. 


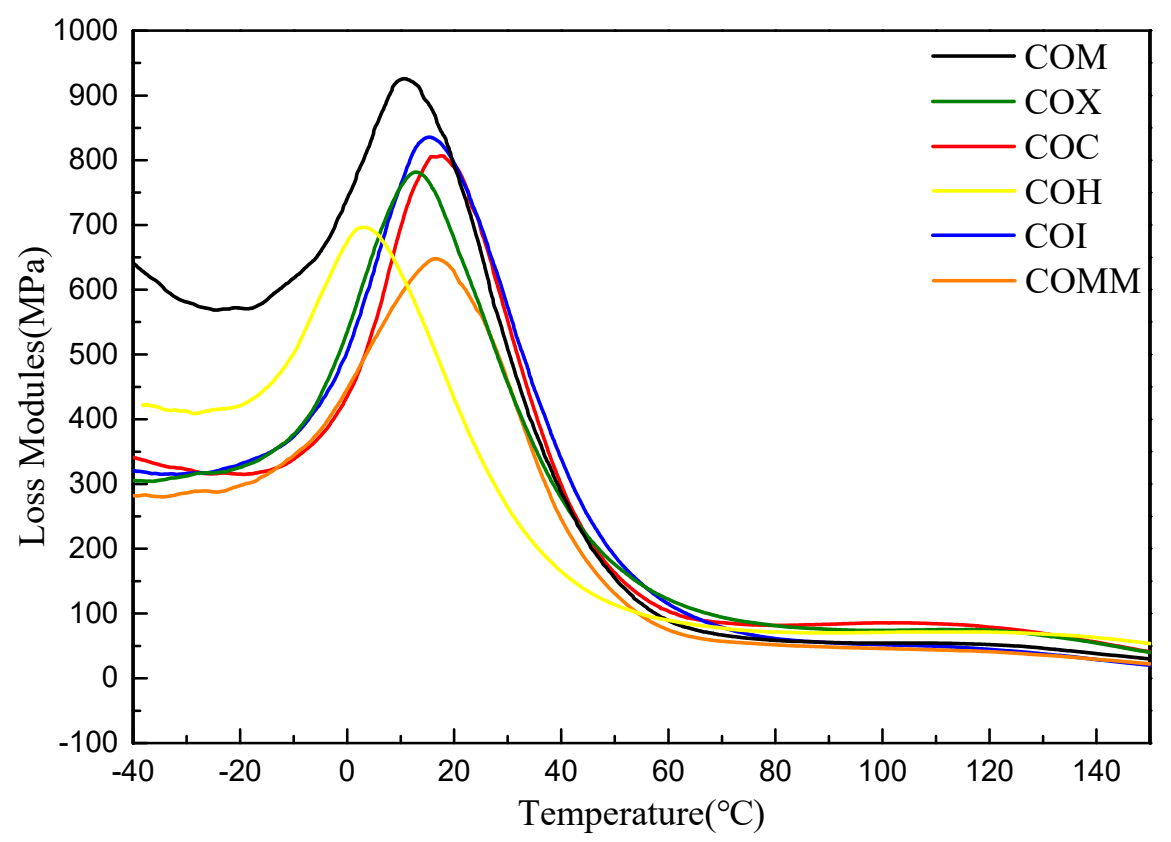

Figure 5. DMA for PA6 containing cyclic compounds.

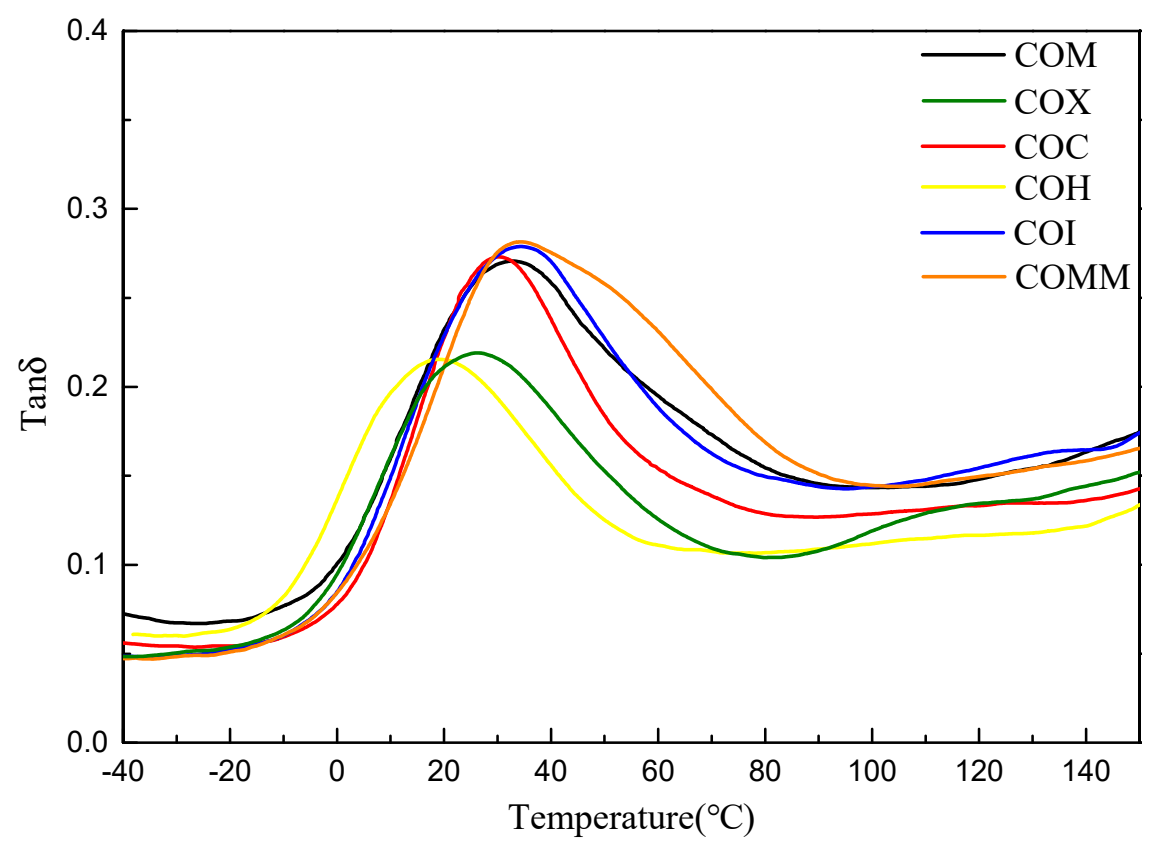

Figure 6. $\tan \delta$ value for PA6 containing cyclic compounds.

\subsubsection{Crystallinity}

The crystal structure of the synthesized product was determined using an X-ray diffractometer at $2 \theta$ angles between $5^{\circ}$ and $45^{\circ}$. Figure 7 shows the XRD spectrum for nylon 6 with different copolymerization components. The figure shows that the amine-containing cycloalkyl group-containing nylon 6 has an $\alpha$-type doublet at $19.5^{\circ}$ and $24^{\circ}$. $\mathrm{COH}$ also shares this feature. Because of the degree of freedom in the torsion for the cycloalkyl group, the crystal form retains a tighter alpha form. However, the COX containing a meta-benzene ring in the amine is crystallized at $21.3^{\circ} \sim 21.5^{\circ}$ and the single peak of the $\gamma$ form is dominant. There is loose $\gamma$ crystallization due to the meta-benzene ring structure, which has less freedom of motion. 


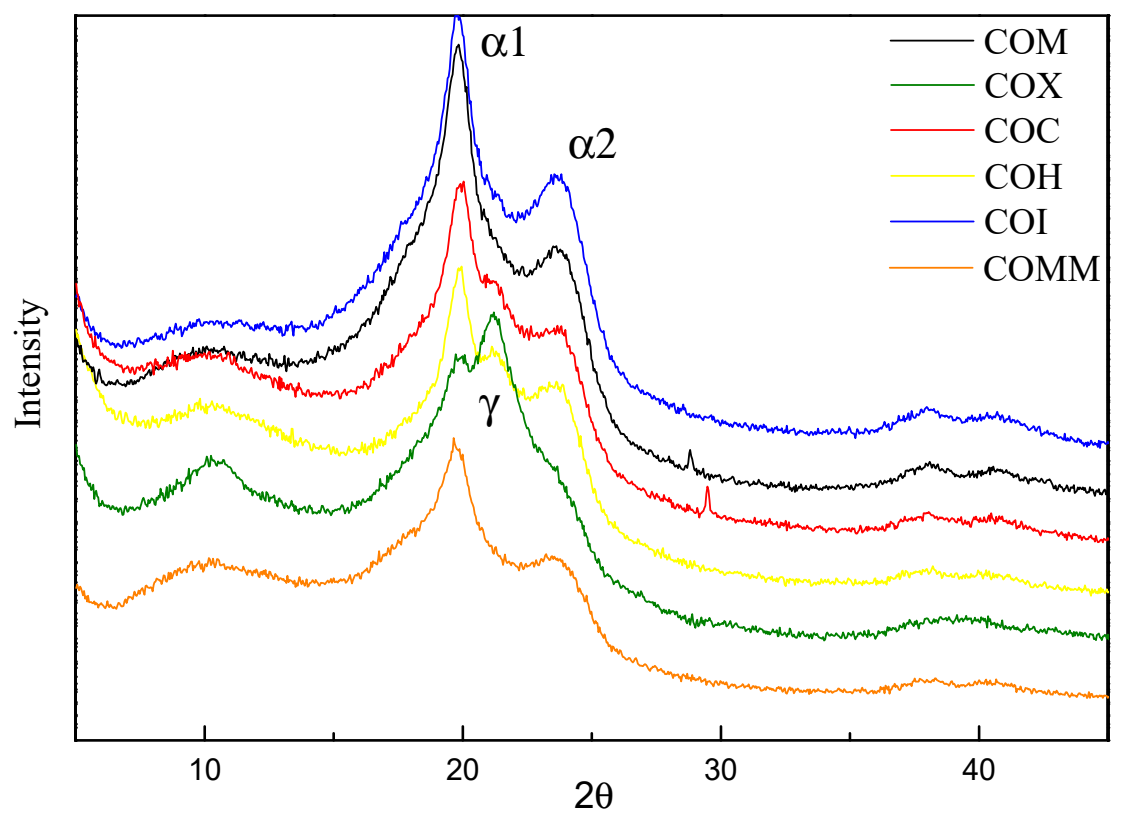

Figure 7. X-ray for PA6 containing cyclic compounds.

\subsubsection{Optical Properties}

Table 3 shows the optical properties and water absorption for nylon 6 with different copolymerization components. The table shows that the nylon 6 copolymerization component can greatly improve the transparency due to the introduction of the bicycloalkyl groups, COMM and COM. It has better transparency and lower haze than monocycloalkane and the meta-benzene ring. The copolymerization of $\mathrm{COH}$ without any cycloalkyl and benzene ring structure results in low transparency and high haze. COMM produces the best transparency and lowest haze because there is minimal crystallization of nylon 6 due to the bulky structure of the dimethyl side chain and bicycloalkane in the structure. COM and COMM have lower water absorption because both contain a bicycloalkyl group and have more hydrophobic carbon chains, so they are more hydrophobic. $\mathrm{COH}$ has the highest water absorption because it is copolymerized without any cycloalkyl and benzene ring structure [8-11].

Table 3. Transparency and water absorption of PA6 containing cyclic compounds.

\begin{tabular}{ccccc}
\hline Polymer & $\begin{array}{c}\text { Transparency } \\
\mathbf{( \% )}\end{array}$ & $\begin{array}{c}\text { Haze } \\
\text { thickness*0.3 } \mathbf{~ m m} \\
\mathbf{( \% )}\end{array}$ & $\begin{array}{c}\text { Water Absorption } \\
\mathbf{2 4} \mathbf{~ h} \mathbf{( \% )}\end{array}$ & $\begin{array}{c}\text { Water Absorption } \\
\mathbf{4 8} \mathbf{~ h} \mathbf{( \% )}\end{array}$ \\
\hline COM & 87.5 & 32.0 & 1.07 & 1.31 \\
COX & 85.8. & 39.4 & 1.51 & 2.13 \\
COC & 87.9 & 52.4 & 1.29 & 1.62 \\
COH & 81.9 & 75.0 & 1.57 & 2.21 \\
COI & 87.3 & 39.4 & 1.30 & 1.62 \\
COMM & 88.8 & 29.2 & 1.24 & 1.42 \\
\hline
\end{tabular}

\subsection{PA6-Containing Dimer Oleic Acid and 4,4'-Methylenebis(2-methylcyclohexylamine)}

These experiments determine the effect of different copolymerization ratios for COMM on PA6. The code for the composition, the composition of the RV and the amine group are shown in Tables 1 and 4 . 
Table 4. Composition of PA6 containing dimer oleic acidand. 4,4'-Methylenebis (2methylcyclohexylamine).

\begin{tabular}{cccccccc}
\hline Polymer & $\begin{array}{c}\text { PA6 } \\
\text { molar ratio }\end{array}$ & $\begin{array}{c}\text { ACID } \\
\text { molar ratio }\end{array}$ & $\begin{array}{c}\text { BASE } \\
\text { molar ratio }\end{array}$ & RV & $\begin{array}{c}\text { NH2 } \\
\text { meq } / \mathbf{k g}\end{array}$ & $\boldsymbol{M}_{\mathbf{w}}$ & $\boldsymbol{M}_{\mathbf{w}} / \boldsymbol{M}_{\mathbf{n}}$ \\
\hline COMM1 & CPL, 98\% & OA, 1\% & MMCA, 1\% & 2.35 & 55 & 38621 & 1.3 \\
COMM3 & CPL, 94\% & OA, 3\% & MMCA, 3\% & 2.28 & 58 & 37552 & 1.45 \\
COMM5 & CPL, 90\% & OA, 5\% & MMCA, 5\% & 2.25 & 61 & 37421 & 1.47 \\
COMM7 & CPL, 86\% & OA, 7\% & MMCA, 7\% & 2.20 & 62 & 35223 & 1.47 \\
COMM10 & CPL, 80\% & OA, 10\% & MMCA, 10\% & 2.13 & 64 & 34226 & 1.49 \\
\hline
\end{tabular}

\subsubsection{Thermal Properties}

Figure 8 shows a TGA diagram of MMCA with different copolymerization ratios under nitrogen gas. COMM1 has the highest value for $T \mathrm{~d} 10$ and $T \mathrm{~d} 50$ are 402 and $444{ }^{\circ} \mathrm{C}$. The $T \mathrm{~d} 10$ and $T \mathrm{~d} 50$ values for each polymer are listed in Table 5.

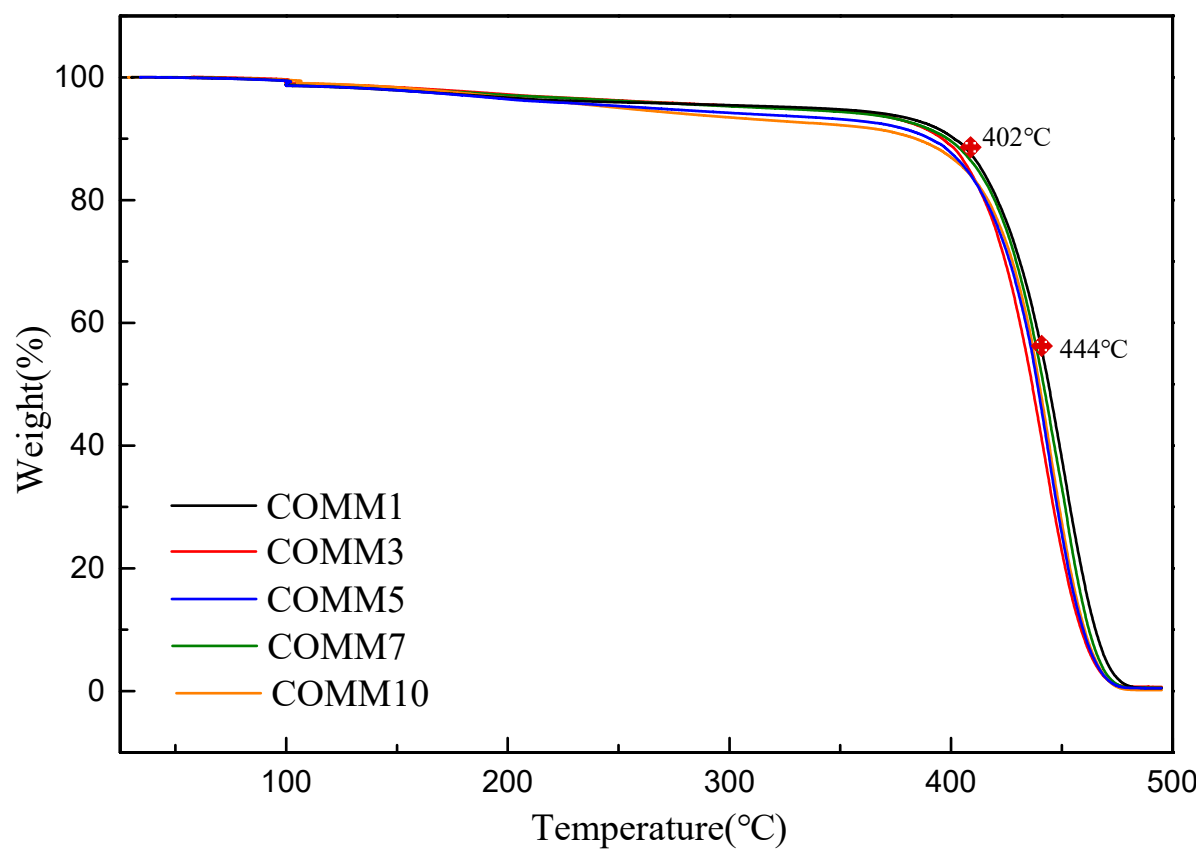

Figure 8. TGA of PA6 containing dimer oleic acid and 4,4'-Methylenebis(2-methylcyclohexylamine).

Table 5. Thermal properties of PA6 containing dimer oleic acid and 4,4'-Methylenebis(2methylcyclohexylamine).

\begin{tabular}{lcccccc}
\hline Polymer & $\begin{array}{c}\boldsymbol{T}_{\mathbf{g}} \\
{ }^{\circ} \mathbf{C}\end{array}$ & $\begin{array}{c}\boldsymbol{T}_{\mathbf{m}} \\
{ }^{\circ} \mathbf{C}\end{array}$ & $\begin{array}{c}\boldsymbol{\Delta H} \\
\mathbf{J} / \mathbf{g}\end{array}$ & $\begin{array}{c}\text { Crystallinity } \\
\mathbf{\%}\end{array}$ & $\begin{array}{c}\mathbf{T d 1 0} \\
{ }^{\circ} \mathbf{C}\end{array}$ & $\begin{array}{c}\mathbf{T d 5 0} \\
{ }^{\circ} \mathbf{C}\end{array}$ \\
\hline COMM1 & 27.7 & 213 & 97.2 & 40.5 & 402 & 444 \\
COMM3 & 34.7 & 207 & 36.1 & 15.0 & 397 & 436 \\
COMM5 & 36.6 & 203 & 33.5 & 13.9 & 387 & 438 \\
COMM7 & 47.7 & 197 & 23.3 & 9.7 & 399 & 441 \\
COMM10 & 34.2 & 191 & 21.6 & 9.0 & 378 & 439 \\
\hline
\end{tabular}

$T_{\mathrm{g}}$ : glass transition measured by DMA, Crystallinity: the value of $100 \%$ crystalline material's melting heat is 240 .

Figure 9 DSC diagram of MMCA with different copolymerization ratios. The figure shows that as the proportion of MMCA increases, the melting peak has a tendency to shift to the left, it is confirmed that the change of its crystal form, when the copolymerization ratio is $1 \%$, it can be observed that the thermal properties of COMM are better than those of pure nylon, and have the best thermal properties. It is known from Table 5 that $\mathrm{Td} 10$ is at $378 \sim 402{ }^{\circ} \mathrm{C}, \mathrm{Td} 50$ is at $436 \sim 444{ }^{\circ} \mathrm{C}$, 
and all polymers have thermal weight loss above $350^{\circ} \mathrm{C}$. Therefore, each polymer has good thermal properties, and as the proportion of copolymerized components increases, the properties of melting point, enthalpy, crystallinity, etc. of COMM decrease, so the copolymerization ratio of $10 \%$ has a lower crystallinity [12-15].

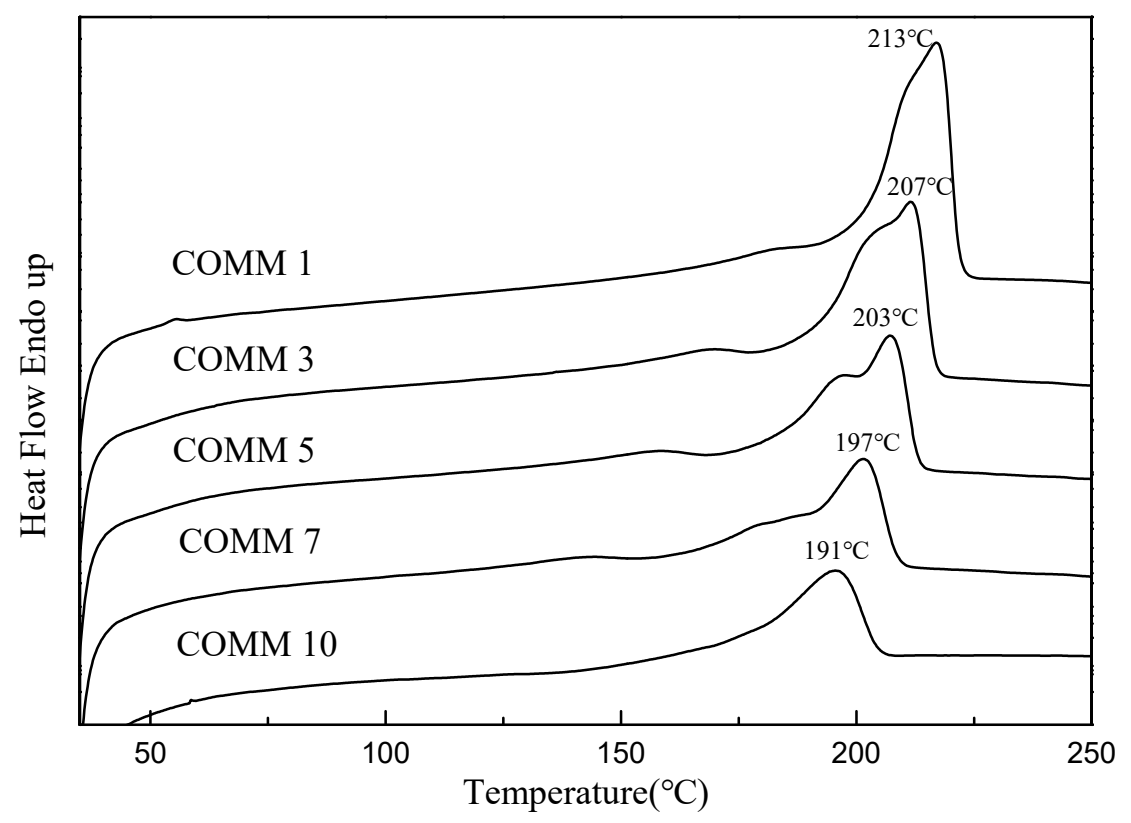

Figure 9. DSC of PA6 containing dimer oleic acid and 4,4'-Methylenebis(2-methylcyclohexylamine).

Figures 10 and 11 show the loss modulus diagram and tan $\delta$ diagram of COMM with different copolymerization ratios. It is known from the figure that the copolymerization ratio of $7 \%$ has the lowest loss modulus, and at $10 \%$ of the copolymerization, the loss modulus increases due to low crystallinity. As the copolymerization ratio increases, the $\tan \delta$ value increases, and the introduction of a long carbon chain gives nylon 6 a higher viscosity $[16,17]$.

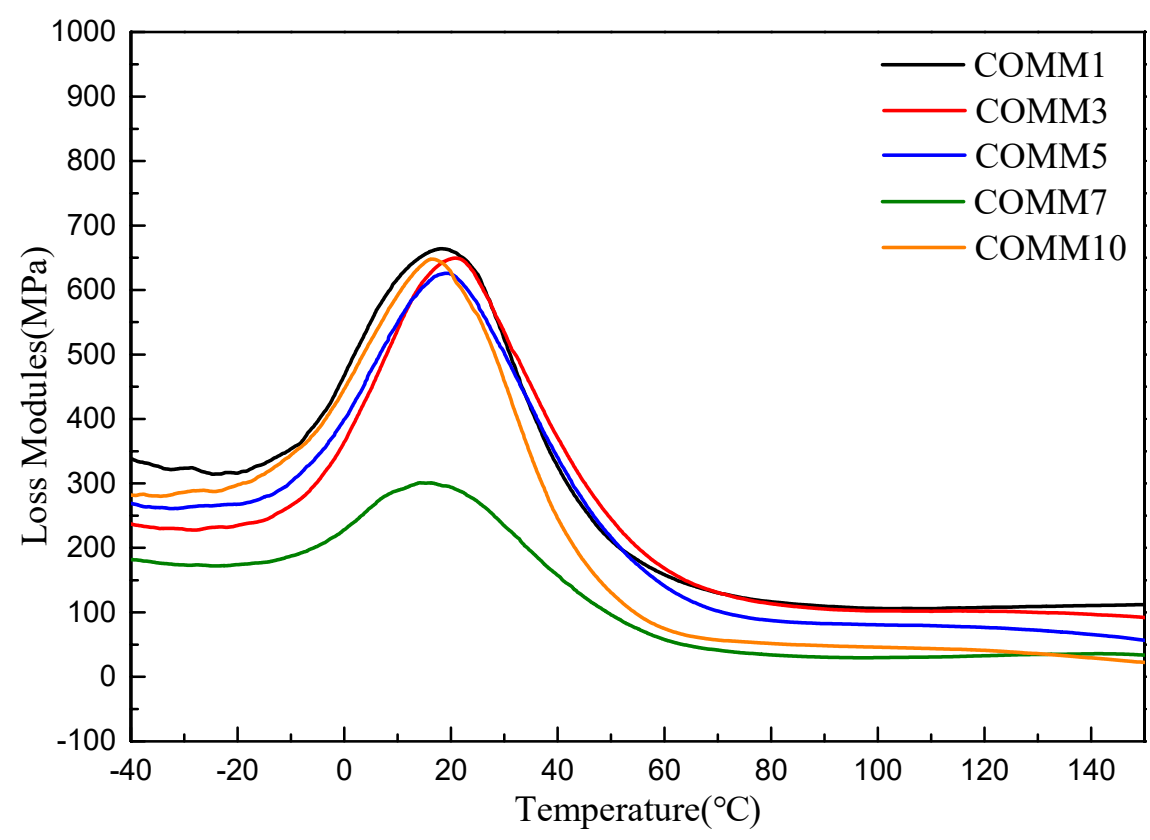

Figure 10. DMA of PA6 containing dimer oleic acid and $4,4^{\prime}$-Methylenebis(2-methylcyclohexylamine). 


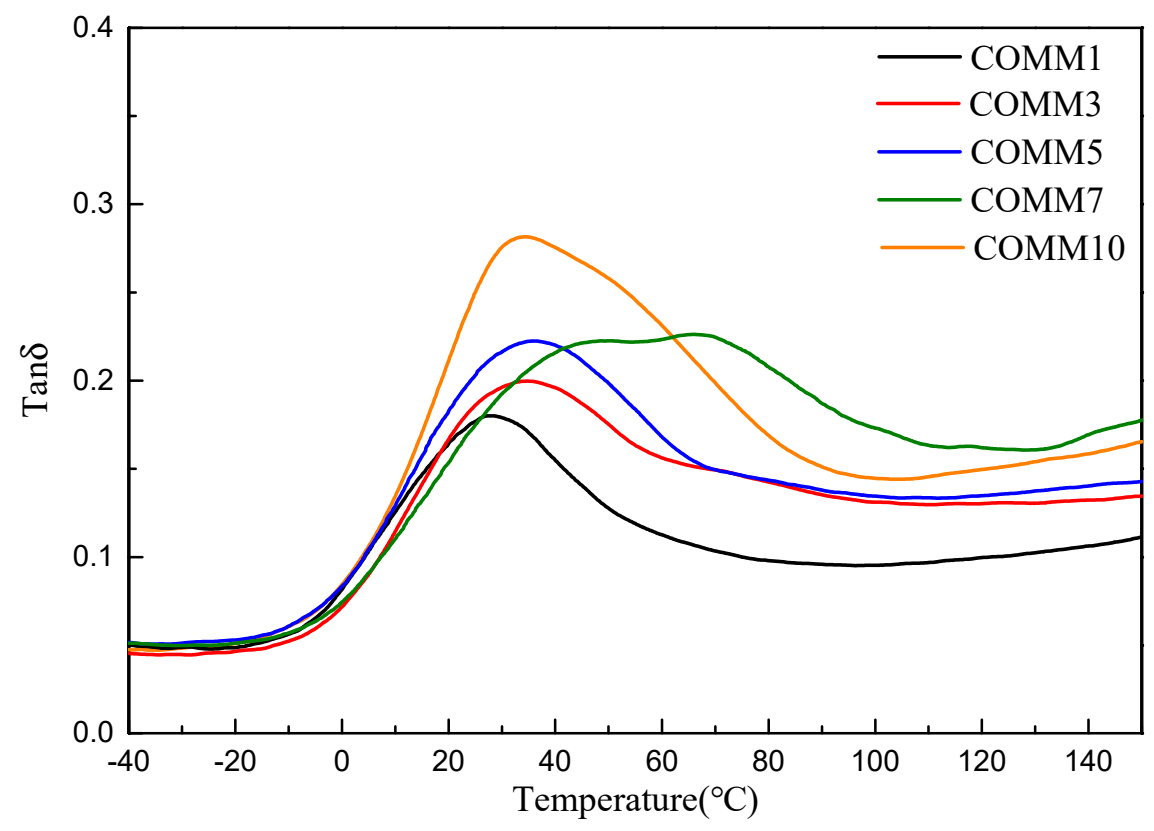

Figure 11. $\tan \delta$ value for PA6 containing dimer oleic acid and 4,4'-Methylenebis(2methylcyclohexylamine.

\subsubsection{Crystallinity}

Figure 12 shows the XRD spectrum for different copolymerization ratios of COMM. It is seen that $1 \%$, $3 \%, 5 \%, 7 \%, 10 \%$ of COMM increases with the proportion of MMCA. Crystallization at $19.5^{\circ}$ and $24.0^{\circ}$ results in an $\alpha$-type doublet. The $\alpha 2$ peak gradually disappears as the proportion of copolymerization increases. As the copolymerization ratio increases, the crystal structure is affected [18-21].

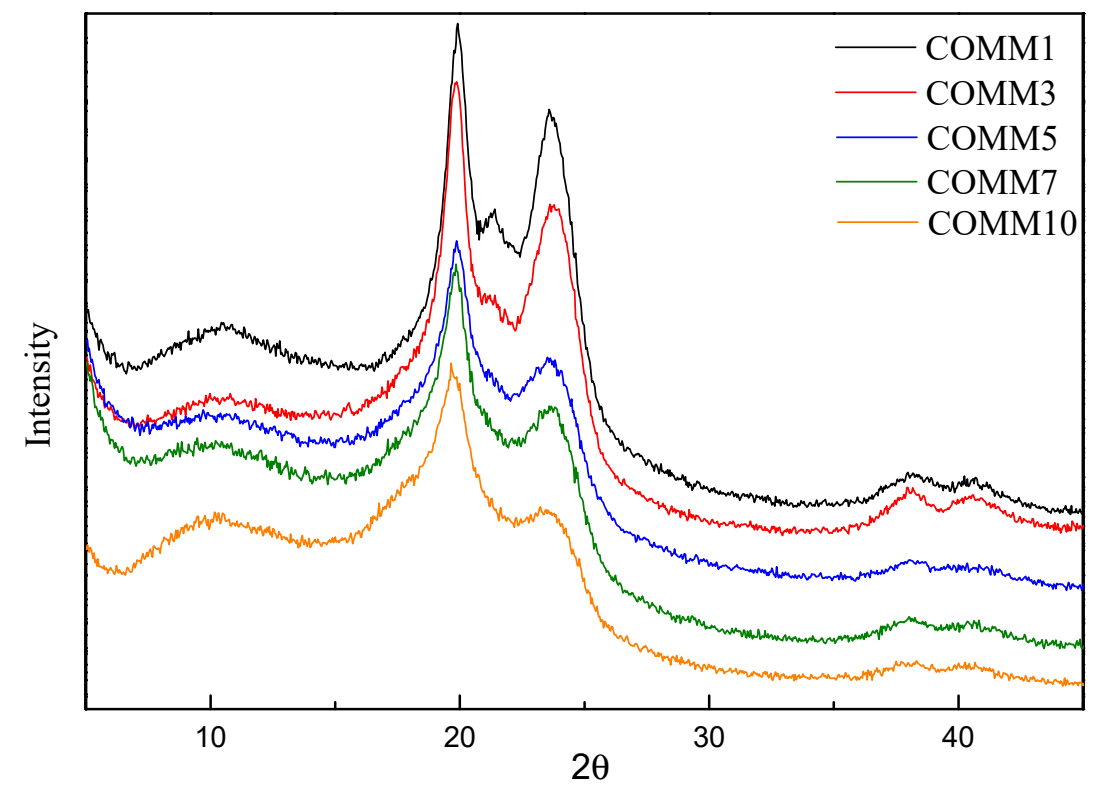

Figure 12. X-ray diffraction spectrum for PA6 containing dimer oleic acid and 4, $4^{\prime}$-Methylenebis(2methylcyclohexylamine).

\subsubsection{Optical Properties}

Table 6 shows the optical properties and water absorption for different copolymerization ratios for COMM. it is seen that as the copolymerization ratio increases, the transparency increases and the haze decreases, mainly due to the increase in the methyl side chain and the cycloalkyl group. COMM 
has the best optical properties when the copolymerized component is $10 \%$. The water absorption decreases as the content of the bicycloalkyl group increases. The lowest water absorption occurs for a copolymerization ratio of $10 \%$ [22-24].

Table 6. Transparency and water absorption for PA6 containing Octadecadienoic acid and 4,4'Methylenebis(2-methylcyclohexylamine).

\begin{tabular}{ccccc}
\hline Polymer & $\begin{array}{c}\text { Transparency } \\
\mathbf{( \% )}\end{array}$ & $\begin{array}{c}\text { Haze } \\
\text { thickness*0.3 } \mathbf{~ m m} \\
\mathbf{( \% )}\end{array}$ & $\begin{array}{c}\text { Water Absorption } \\
\mathbf{2 4} \mathbf{h} \mathbf{( \% )}\end{array}$ & $\begin{array}{c}\text { Water Absorption } \\
\mathbf{4 8} \mathbf{~ h} \mathbf{( \% )}\end{array}$ \\
\hline COMM1 & 65.8 & 89.6 & 2.35 & 2.84 \\
COMM3 & 76.9 & 84.6 & 1.78 & 2.61 \\
COMM5 & 84.7 & 73.5 & 1.52 & 2.32 \\
COMM7 & 87.1 & 41.9 & 1.22 & 1.84 \\
COMM10 & 88.8 & 29.2 & 1.24 & 1.42 \\
\hline
\end{tabular}

\section{Conclusions}

A ring-opening polymerization reaction for caprolactam is used and a dimer acid is copolymerized with different structural amines, to produce a series of non-crystalline nylon 6 . Amine 6 containing a cycloalkyl group a crystal that is mainly in the form of $\alpha$, which is also the case for $\mathrm{COH}$. COX containing a meta-benzene ring in the amine is mainly in the $\gamma$ form. Different copolymerized nylon 6 COMM has the lowest crystallinity. COM containing bicycloalkyl has a higher loss modulus than other nylon 6 containing methyl and benzene rings. COMM and COI containing a methyl group have a greater influence on nylon 6 and are very rigid, so the value for $T_{\mathrm{g}}$ is higher. The introduction of nylon 6 copolymerization component into COMM and COM containing bicycloalkyl groups can greatly improve the transparency, so it has better transparency and low haze than monocycloalkane and metacyclic benzene ring.

In the second part, different ratios of MMCA and dimerized oleic acid were prepared to synthesize different proportions of amorphous nylon 6 to investigate the effect of different ratios on the properties of nylon 6 . Crystal structure at both $19.5^{\circ}$ and $24.0^{\circ}$ crystals showed an $\alpha$-type doublet, and its peak gradually shifted from $\alpha$-type to $\gamma$-type as the copolymerization ratio increased, confirming the influence on crystal structure. With regard to the thermal properties, the melting point, enthalpy, crystallinity, and other properties of COMM decrease with the increase of the proportion of copolymerization components. The optimal optical properties are obtained when the copolymerization component is $10 \%$.

Author Contributions: C.-C.L. supervised the experiments, reviewed and revised the manuscript. C.-N.H. analyzed the results and wrote the manuscript. C.-M.W. planned the experiments and took an active role in the preparation of the manuscript. H.-W.L. performed the experiments and analyzed the results. W.-F.T., L.-C.L. and C.-M.C. contributed to the interpretation of data concerning TOC properties. All authors have read and approved the final manuscript.

Acknowledgments: The Ministry of Science and Technology of Taiwan, ROC, financially supported part of this work, under contract numbers: MOST 107-2218-E-011-026.

Conflicts of Interest: The authors declare no conflict of interest.

\section{References}

1. Kohan, M.I. Nylon Plastics Handbook; Hanser Publishers: Munich, Germany, 1995; p. 377.

2. Aharoni, S.M. n-Nylons: Their Synthesis, Structure, and Properties; Wiley: Hoboken, NJ, USA, 1997; p. 170.

3. Dolden, J.G. Structure-property relationships in amorphous polyamides. Polymer 1976, 17, 875-892. [CrossRef]

4. Lai, C.C.; Chen, S.Y.; Chen, M.H.; Chen, H.L.; Hsiao, H.T.; Liu, L.C.; Chen, C.M. Preparation and characterization of heterocyclic polyamide 6 (PA 6) with high transparencies and low hygroscopicities. J. Mol. Str. 2019, 1175, 836-843. [CrossRef] 
5. Androsch, R.; Stolp, M.; Radusch, H.J. Crystallization of amorphous polyamides from the glassy state. Acta Polym. 1996, 47, 99-104. [CrossRef]

6. Van Velthoven, J.L.; Gootjes, L.; Noordover, B.A.; Meuldijk, J. Bio-based, amorphous polyamides amorphous with tunable thermal properties. Eur. Polym. J. 2015, 66, 57-66. [CrossRef]

7. Zheng, J.; Siegel, R.W.; Toney, C.G. Polymer crystalline structure and morphology changes in nylon-6/ZnO nanocomposites. J. Polym. Sci. Part B 2003, 41, 1033-1050. [CrossRef]

8. Li, P.H.; Wang, C.Y.; Li, G.; Jiang, J.M. Highly organosoluble and transparent polyamides containing cyclohexane and trifluoromethyl moieties: Synthesis and characterization. Polym. Lett. 2009, 3, 703-712. [CrossRef]

9. Javadi, A.; Shockravi, A.; Koohgard, M.; Malek, A.; Shourkaei, F.A.; Ando, S. Nitro-substituted polyamides: A new class of transparent and highly refractive materials. Eur. Polym. J. 2015, 66, 328-341. [CrossRef]

10. Li, Y.; Goddard III, W.A. Nylon 6 crystal structures, folds, and lamellae from theory. Macromolecules 2002, 35, 8440-8455. [CrossRef]

11. Rusua, G.; Uedab, K.; Rusua, E.; Rusuc, M. Polyamides from lactams by centrifugal molding via anionic ring-opening polymerization. Polymer 2001, 42, 5669-5678. [CrossRef]

12. Guo, D.D.; Sheng, S.R.I.; Sang, X.Y.; Huang, Z.Z.; Liu, X.L. New Organosoluble and Optically Transparent Polyamides Containing Xanthene Units and Methyl Pendant Groups From 9,9-Bis[4-(4-carboxyphenoxy)-3methylphenyl]xanthene. J. Macromol. Sci. Part A 2015, 52, 950-959. [CrossRef]

13. Kiani, H.; Nasef, M.M.; Javadi, A.; Lotf, E.A.; Nemati, F.J. Highly refractive, transparent, and solution processable polyamides based on a noncoplanar ortho-substituted sulfonyl-bridged diacid monomer containing chlorine side groups. Polym. Res. 2013, 20, 2-12. [CrossRef]

14. Rajesh, J.J.; Bijwe, J. Effect of water absorption on erosive wear behaviour of polyamides. J. Mater. Sci. 2002, 37, 5107-5113. [CrossRef]

15. Shidara, Y.; Yunoki, T.; Miura, S.; Shibasaki, Y.; Fujimori, A. Effect of the isothermal crystallization method on amorphous block copolymers of aromatic polyamides and their packing behavior in two-dimensional films for screening of potential crystallization ability. Polym. Eng. Sci. 2018, 58, 2019-2030. [CrossRef]

16. Lee, W.L.; Lin, J.S.; Juang, F.S.; Chen, H.F.; Chen, C.M.; Liu, L.C. Manufacture of optical substrates with high gas resistance as well as antistatic capability and their applications for flexible organic light emitting diodes (flexible OLEDs). Mater. Chem. Phys. 2013, 142, 701-706. [CrossRef]

17. Kolesov, I.; Androsch, R. The rigid amorphous fraction of cold-crystallized polyamide 6. Polymer 2012, 53, 4770-4777. [CrossRef]

18. López-Rubio, A.; Gavara, R.; Lagarón, J.M. Unexpected partial crystallization of an amorphous polyamide as induced by combined temperature and humidity. J. Appl. Polym. Sci. 2006, 102, 1516-1523. [CrossRef]

19. Rwei, S.P.; Tseng, Y.C.; Chiu, K.C.; Chang, S.M.; Chen, Y.M. The crystallization kinetics of Nylon 6/6T and Nylon 66/6T copolymers. Thermochim. Acta 2013, 555, 37-45. [CrossRef]

20. Maskus, P.P. In search of excellence : ANTEC ' 91 Conference Proceedings. In Proceedings of the Annual Technical Conference-ANTEC, Montreal, QC, Canada, 5-9 May 1991; Volume 37, pp. 1385-1388.

21. Hernandez, R.J.; Giacin, J.R.; Grulke, E.A. The sorption of water vapor by an amorphous polyamide. J. Membr. Sci. 1992, 65, 187-199. [CrossRef]

22. Srinivasan, R.; McGrath, J.E. Synthesis of Novel Monomers for Cyanate Ester Matrices. Am. Chem. Soc. Polym. Prepr. Div. Polym. Chem. 1992, 33, 225-232.

23. Kuo, C.C.; Liu, L.C.; Liang, W.C.; Liu, H.C.; Chen, C.M. Preparation of polylactic acid (PLA) foams with supercritical carbon dioxide and their applications for reflectors of white light-emitting diode (LED) lamps. Mater. Res. Bull. 2015, 67, 170-175. [CrossRef]

24. Kuo, C.C.; Liu, L.C.; Liang, W.C.; Liu, H.C.; Chen, C.M. Preparation of polypropylene (PP) composite foams with high impact strengths by supercritical carbon dioxide and their feasible evaluation for electronic packages. Compos. Part B 2015, 79, 1-5. [CrossRef]

(C) 2019 by the authors. Licensee MDPI, Basel, Switzerland. This article is an open access article distributed under the terms and conditions of the Creative Commons Attribution (CC BY) license (http:/ / creativecommons.org/licenses/by/4.0/). 